\title{
La teoría de Vygostky y el pensamiento crítico en los docentes del Instituto Superior Pedagógico de Huancavelica
}

\section{The theory of Vygotsky and critical thinking on teachers the Higher Teaching Institute of Huancavelica}

\author{
Zoraida Rocío Manrique Chávez
}

Universidad Nacional de Huancavelica, Jr. Victoria Garma 275, Huancavelica, Perú

DOI: https://doi.org/10.33017/RevECIPeru2013.0013/

\section{Resumen}

En la formación magisterial, una de las capacidades básicas que debería tener el docente para cumplir con los roles que se le asigna es saber aprender, pues no podría enseñar a "aprender a aprender" si el mismo no posee esta habilidad. En este marco, se requiere que el docente circule dentro de un paradigma de conceptualización, aplicación, análisis, sintetización y evaluación de información procedente de su observación, experiencia, reflexión, razonamiento o comunicación que le permita desenvolverse adecuadamente dentro de su contexto social y cultural. Lev S. Vygostky plantea que los procesos psicológicos son cambiantes y dependen en gran medida del entorno vital. Por lo cual, los desarrollos de las personas únicamente pueden ser explicados en términos de la interacción social. El pensamiento crítico se propone analizar o evaluar la estructura y consistencia de los razonamientos, particularmente opiniones o afirmaciones que el docente acepta como verdaderas en el contexto de la vida socio cultural. En tal sentido, ésta investigación surge con el propósito de determinar el nivel de pensamiento crítico que se manifiesta en los docentes del Instituto Superior Pedagógico Público de Huancavelica en relación a las características de la teoría de Vygotsky. Para alcanzar este objetivo se realizó un estudio descriptivo con 8 docentes de dicha institución; con un diseño de un grupo sólo después, a los cuales se les aplicó dos intrumentos: una encuesta de autoevaluación y ficha de observación de videos de sesiones didácticas elaboradas en base a los estándares y estrategias intra e interpsicológicas propuestos en la teoría de Vigotsky. Los resultados obtenidos del análisis de los datos obtenidos muestran que todos los docentes presentan un nivel de pensamiento crítico por encima de aceptable. Además, se obtuvo un coeficiente de correlación de Pearson de $\mathrm{r}_{\mathrm{XY}}=0,73$ en cuanto a los resultados en ambos instrumentos, validado por la prueba $\mathrm{T}$ de Studens con un nivel de significancia del 0.05 y un coeficiente de proporcionalidad de variabilidad compartida o explicada del $53 \%$. En esta investigación se pone en evidencia y nos permite reflexionar que en la práctica pedagógica, los maestros deberán de cambiar radicalmente su relación con el conocimiento que tendrá que ser activa y crítica y deberá percibirse a sí mismos y actuar como creadores y no sólo transmisores de conocimientos.

Descriptores: Teoría de Vygotsky, pensamiento crítico, autoevaluación

\section{Abstract}

In teacher education, one of the basic skills that the teacher should have to meet the roles assigned to it is knowing how to learn, as I could not teach "learning to learn " if it does not have this ability. In this context, the teacher is required to circulate within a paradigm of conceptualization, application, analysis, synthesis and evaluation of information from observation, experience, reflection, reasoning, or communication that allows deal appropriately within their social and cultural context. Lev S. Vygotsky argues that psychological processes are changing and depend largely on the living environment. Therefore, the development of individuals can only 
be explained in terms of social interaction. Critical thinking is to analyze and evaluate the structure and consistency of reasoning, particularly opinions or assertions that the teacher accepts as true in the context of socio-cultural life. In this sense, this research began with the purpose of determining the level of critical thinking that is manifested in teachers Public Pedagogical Institute of Huancavelica in relation to the characteristics of Vygotsky's theory. To achieve this objective, a descriptive study with 8 teachers from the institution, with a design only after a group, to which they applied two instruments: a survey of self- observation and record videos made based didactic sessions to the standards and strategies proposed intra and interpsychological Vygotsky 's theory. The results obtained from the analysis of the data obtained show that all teachers have a critical thinking level above acceptable. In addition, we obtained a Pearson correlation coefficient of $r_{X Y}=0,73$ the results in terms of both instruments, validated by studens $t$ test with a significance level of 0.05 and a coefficient of proportionality of shared or explained variability $53 \%$. This research highlights and allows us to reflect that in teaching practice, teachers should radically change their relationship with the knowledge that will need to be active, critical and should perceive themselves and act not only as creators and transmitters knowledge.

\section{Keywords: Theory of Vygotsky, critical thinking, autoevaluation.}

\section{Introducción}

En esta última década, la educación latinoamericana ha dado un paso trascendental en el ámbito de la psicología educativa y científica. Luego de un extenso periodo de dominación del paradigma conductista y el posterior neoconductismo, empieza a ser gratamente evidente la presencia del paradigma emergente cuya denominación es cognitivo, ecológico y contextual. La otra tendencia es que las instituciones de educación superior se centran, muchas veces, en la transmisión de conocimientos ya elaborados; en simples procesos de información o preparación de tipo técnica, cuyo principal objetivo es la producción de un profesional para cubrir la demanda de un mercado laboral; olvidando que la educación es un proceso de formación, de acceso al pensamiento crítico y a la construcción del saber.

Dentro del marco de este paradigma emergente, se requiere que el docente muestre en su accionar dentro del aula características que tengan que ver con la interpretación, análisis, evaluación e inferencia; como también la explicación de las consideraciones de evidencia, conceptuales, metodológicas, criteriológicas o contextuales en las cuales basa su juicio, argumento o forma de actuar en el proceso de enseñanza - aprendizaje. [1]

Christian Miranda en su estudio sobre el Pensamiento Crítico en Docentes de Educación General Básica en Chile plantea que a partir del mejoramiento del pensamiento crítico hay un progreso significativo, hay una avance en la capacidad de formular hipótesis y estrategias para el análisis de datos; aplicar técnicas, modelos y reglas para la solución de problemas; demostrar espacio, flexibilidad y creatividad; evaluar conjeturas mediante el razonamiento, encontrar relaciones y sacar conclusiones, presentándose una aumento significativo en la comunicación así como un aumento significativo, siendo evidente su capacidad para elegir, organizar y aplicar esquemas apropiados, redactar eficazmente, comunicar información cuantitativa y cualitativa a partir de contenidos.

Lev S. Vygotsky plantea que es fundamental considerar al individuo como el resultado del proceso histórico y social donde se encuentra, donde el lenguaje desempeña un papel esencial para conocer las condiciones socioculturales [2]

En este sentido; describir, analizar y establecer la relación entre los postulados de la teoría de Vygotsky y los planteamientos del pensamiento crítico en los docentes del Instituto Superior Pedagógico Público de Huancavelica resulta importante, ya que a partir de ello no sólo se podrá definir criterios para impartir una formación profesional en un marco de superación acorde con la nueva era del conocimiento, sino también se tendrá un impacto favorable en el desarrollo integral y sostenido que tanto necesita la región de Huancavelica. En tal sentido, el problema motivo de estudio se formuló en los términos siguientes: ¿Cuál es la relación entre el pensamiento crítico que se manifiesta en los docentes del Instituto Superior Pedagógico Público de Huancavelica y las características de la teoría de Vygotsky? Como objetivo principal del estudio se planteó: Determinar el nivel de relación entre el pensamiento crítico que se manifiesta en los docentes del Instituto Superior Pedagógico Público de Huancavelica y las características de la teoría de Vygotsky 


\subsection{Modelo de Aprendizaje Sociocultural}

En este modelo se sostiene que los procesos, desarrollo y aprendizaje, interactúan entre sí, considerando el aprendizaje como un factor del desarrollo. Además, la adquisición de aprendizaje se explica como formas de socialización. Concibe al hombre como una construcción más social que biológica, en donde las funciones superiores son fruto del desarrollo cultural e implican el uso de mediadores. Esta estrecha relación entre desarrollo y aprendizaje que Vygotsky destaca, lo lleva a formular su famosa teoría de la "Zona de Desarrollo Próximo" (ZDP). Esto significa, en palabras del mismo Vygotsky: "la distancia entre el nivel de desarrollo, determinado por la capacidad para resolver independientemente un problema, y el nivel de desarrollo potencial, determinado a través de la resolución de un problema bajo la guía de un adulto o en colaboración con otro compañero más capaz".

La zona de desarrollo potencial estaría, así, referida a las funciones que no han madurado completamente en el discente, pero que están en proceso de hacerlo. De todos modos, subraya que el motor del aprendizaje es siempre la actividad del sujeto, condicionada por dos tipos de mediadores: "herramientas" y "símbolos", ya sea autónomamente en la "zona de desarrollo real", o ayudado por la mediación en la "zona de desarrollo potencial". Las "herramientas" son las expectativas y conocimientos previos del discente que transforman los estímulos informativos que le llegan del contexto.

Los "símbolos" (herramientas psicológicas) son el conjunto de signos que utiliza el mismo sujeto para hacer propios dichos estímulos. Modifican no los estímulos en sí mismo, sino las estructuras de conocimiento cuando aquellos estímulos se interiorizan y se convierten en propios. De este modo, lo que separa las funciones psicológicas elementales de las superiores, es que las segundas usan signos que actúan como mediadores, con lo que el control pasa del contexto social al individuo, permitiéndole, por tanto, anticipar y planificar su acción. Al decir que la acción del hombre está mediada, Vygotsky se refiere a que los sistemas de signos, además de permitir una interpretación y el control de la acción social, se vuelven mediadores de la propia conducta individual.

Todo este proceso recibe el nombre de "ley de la doble formación" puesto que el conocimiento se adquiere procesándolo, primero, desde el exterior, con las "herramientas" y reestructurándolo luego en el interior, a través de los "símbolos". Los conocimientos estructurados con ayuda de los mediadores ("herramientas" y "símbolos") generan en el discente la mencionada "zona de desarrollo potencial" que le permite acceder a nuevos aprendizajes, creándose así un cierto grado de autonomía e independencia para aprender a aprender más [3].

En el aprendizaje, la actividad del discente está mediada por la actividad del docente, que es el que debe ayudarle a activar los conocimientos previos (a través de las "herramientas") y a estructurar los conocimientos previos (a través de los "símbolos") proponiéndole experiencias de aprendizaje ni demasiado fáciles ni demasiado difíciles, sino en el límite de las posibilidades del sujeto. Es decir, en su "área o zona de desarrollo potencial" con el fin de ir ampliándola y desarrollándola.

\subsection{Modelo de Pensamiento Crítico}

El desarrollo del pensamiento que aspiramos a promover es un proceso que la persona tiene que asumir y dirigir a partir de su potencial. La base para ello es la actividad de estudio y el proceso de aprendizaje que ella implica. En última instancia, el currículo es una estrategia para promover un proceso de aprendizaje que sea continuo y acumulativo y que se traduzca en desarrollo humano, es decir, en modificación y enriquecimiento permanente o duradero de la persona. Para que el aprendizaje tenga este efecto sobre el desarrollo, es necesario sustituir el pseudo-aprendizaje, que aún predomina en nuestros salones de clases, por el aprendizaje auténtico [4].

Es necesario pensar, con libertad y realismo, en el maestro que requerimos y deseamos para la educación básica en el Perú. No quiere ser este un ejercicio futurista sino el esbozo de una propuesta de maestro que, en el marco de un proyecto cultural y educativo para el país, sea punto de referencia para la formulación de una política integral de desarrollo del magisterio peruano. El maestro del futuro tendrá que desaprender y volver a aprender continuamente y aceptar que no lo sabe todo. Por lo expuesto: "Los maestros tendrán que cambiar radicalmente su relación con el conocimiento que tendrá que ser activa y crítica y deberá percibirse a sí mismos y actuar como creadores y no sólo transmisores de conocimientos".

En teoría, para poder ser un buen pensador crítico se debería seguir y desarrollar los estándares intelectuales $y$ las estrategias intra e interpsicológicos. Existen estándares intelectuales 
universales que deben aplicarse al pensamiento cada vez que se quiera evaluar la calidad del razonamiento sobre un problema, un tema o una situación. Pensar críticamente implica dominar estos estándares. Para ayudar a que los discentes los aprendan, los docentes deben plantear preguntas que exploren el pensamiento del estudiante, preguntas que responsabilicen al estudiante por su proceso de pensamiento, preguntas que, por su uso consistente por parte del maestro en la clase, sean interiorizadas por los estudiantes como preguntas que deben plantearse ellos mismos. Aunque existen un gran número de estándares universales, los siguientes son los más significativos: claridad, exactitud, precisión, pertinencia, profundidad, amplitud y lógica. Así como las estrategias intra e interpsicológicas, las cuales son: Humildad intelectual, coraje o entereza intelectual, empatía intelectual, autonomía intelectual, integridad intelectual, perseverancia intelectual y confianza en la razón [5].

\section{Metodología}

El método que se empleó fue el descriptivo, y el diseño de un grupo sólo después para una muestra de 8 docentes del Instituto Superior Pedagógico Público de Huancavelica. Entre los materiales, se emplearon una encuesta de autoevaluación y una ficha de observación de los videos de las sesiones didácticas de los docentes, cuyos resultados se evaluaron a través de técnicas estadísticas que permitieron realizar el tratamiento de los datos y su comprobación de la hipótesis a través de la prueba $T$ de Student y el manejo del paquete estadístico del SPSS

\section{Resultados y discusión}

En cuanto a la encuesta de autoevaluación:

Tabla N 1: Categorización de los resultados obtenidos de la ficha de autoevaluación

\begin{tabular}{|l|c|c|}
\hline \multicolumn{1}{|c|}{ CATEGORÍAS } & fi & fi\% \\
\hline Pensamiento crítico óptimo & 2 & 25 \\
\hline Pensamiento crítico bueno & 6 & 75 \\
\hline Pensamiento crítico aceptable & 0 & 0 \\
\hline Pensamiento crítico poco adecuado & 0 & 0 \\
\hline Pensamiento crítico inadecuado & 0 & 0 \\
\hline
\end{tabular}

El $25 \%$ de los docentes presentan un pensamiento crítico óptimo, mientras que el $75 \%$ de los docentes presenta un pensamiento crítico bueno. Todos los docentes manifiestan un pensamiento crítico por encima del nivel aceptable.
En cuanto a la observación de los videos de las sesiones didácticas:

Tabla N²: Categorización de los resultados obtenidos de la ficha de observación de videos

\begin{tabular}{|l|c|c|}
\hline \multicolumn{1}{|c|}{ CATEGORÍAS } & $\mathrm{fi}$ & $\%$ \\
\hline Pensamiento crítico óptimo & 0 & 0 \\
\hline Pensamiento crítico bueno & 2 & 25 \\
\hline Pensamiento crítico aceptable & 6 & 75 \\
\hline Pensamiento crítico poco adecuado & 0 & 0 \\
\hline Pensamiento crítico inadecuado & 0 & 0 \\
\hline
\end{tabular}

El $25 \%$ de los docentes evidencian en el desarrollo de sus sesiones didácticas un pensamiento crítico bueno, mientras que el $75 \%$ de los docentes presenta un pensamiento crítico aceptable. En el desarrollo de sus sesiones didácticas, todos los docentes manifiestan un pensamiento crítico por encima del nivel aceptable.

Aplicando el coeficiente de correlación de Pearson se obtuvo que: $r_{X Y}=0,73$, lo cual nos indica que existe una relación directa entre los resultados obtenidos en la ficha de autoevaluación y los obtenidos en la ficha de observación de los videos. Para determinar el nivel de significación de este coeficiente, usamos la prueba de "T" de Studens con un nivel de significancia de 0,05 , obteniendo que la correlación encontrada no proviene de una población cuya correlación es nula. Esto nos indica que efectivamente existe relación entre los puntajes obtenidos de la ficha de autoevaluación y la ficha de observación de los videos. Además, a través del Coeficiente de proporcionalidad de variabilidad compartida o explicada: $\mathrm{r}_{\mathrm{XY}}^{2}$, encontramos que el $53 \%$ de los valores obtenidos en la ficha de observación de los videos se pueden explicar a través de los resultados obtenidos en la encuesta de autoevaluación.

Se ha encontrado que las características de la teoría de Vygotsky que se manifiestan en los docentes son el contexto histórico-cultural, la interacción social y el uso de las herramientas psicológicas. Además, las categorías que se manifiestan en el pensamiento crítico de los docentes son los estándares intelectuales, los elementos del pensamiento $y$ las destrezas intra e interpsicológicas.

También, las características de la teoría de Vygotsky que se manifiestan en los docentes y las categorías que se manifiestan en el pensamiento crítico se encuentran relacionadas directamente, tanto en la 
encuesta de autoevaluación donde se encontró un coeficiente de correlación de Pearson fue: $r_{X Y}=0,73$ con un nivel de significancia de $\alpha=0,05$, así como en la observación de los videos de las sesiones didácticas donde el coeficiente de correlación de Pearson fue: $r_{X Y}=0,78$ con un nivel de significancia de $\alpha=0,05$.

\section{Discusión de los resultados}

Se aplicaron dos instrumentos que nos permitieron conocer el nivel de pensamiento crítico de los docentes que formaron parte de nuestra muestra de estudio.

El primer instrumento fue una encuesta de autoevaluación donde se constata que el $75 \%$ de los docentes poseen un pensamiento crítico bueno y el $25 \%$ poseen un pensamiento crítico óptimo. Todos los docentes de la muestra evidencian un pensamiento crítico por encima de la categoría aceptable.

Por otro lado, el segundo instrumento fue una ficha de observación de video de una sesión didáctica de los docentes. Los resultados obtenidos reflejan que el $25 \%$ de los docentes poseen un pensamiento crítico bueno y el $75 \%$ poseen un pensamiento crítico aceptable. Todos los docentes encuestados evidencian un pensamiento crítico más que aceptable.

La aplicación del coeficiente de correlación de Pearson nos ha permitido encontrar que entre los resultados obtenidos en la ficha de autoevalución y los obtenidos por medio de la ficha de observación de los videos de las sesiones didácticas existe una relación directa determinada por el valor de $\mathrm{r}_{\mathrm{XY}}=0,73$.

La aplicación de la Prueba T de Studens con un nivel de significancia de 0.05 , nos muestra que esta relación no proviene de una población donde la correlación es nula. Además el coeficiente de proporcionalidad de variabilidad compartida $r_{X Y}^{2}=0.53$ pone de manifiesto que un $53 \%$ de los resultados obtenidos en la observación de los videos pueden ser explicados según los resultados obtenidos en la ficha de autoevaluación. Esto pone de manifiesto que el nivel de pensamiento crítico de los docentes que formaron parte de este estudio es por lo menos aceptable, tanto en su manifestación personal como en el momento de llevar a cabo una sesión didáctica.
La elección de estrategias demanda del docente la capacidad de reflexión para hacer la mejor elección en base a su análisis del contexto en el cual se va a realizar las actividades didácticas. Vygotsky plantea que la enseñanza debe ser activa, estratégica consciente, automatizada y reflexiva.

Lo fundamental del enfoque de Vygotsky consiste en considerar al individuo como el resultado del proceso histórico y social donde el lenguaje desempeña un papel esencial para conocer las condiciones socioculturales. Los resultados encontrados en los ítems relacionados a la encuesta de autoevaluación y en la observación de los videos de las sesiones didácticas reflejan que en altos porcentajes los docentes consideran el contexto socio cultural a partir de un ambiente motivador, donde existan condiciones sociales adecuadas con sus respectivas características socioculturales y demostrando responsabilidad tanto en el desarrollo de sus labores académicas como en actividades administrativas.

Vygotsky también plantea que el nivel de desarrollo y aprendizaje que el individuo puede alcanzar con la ayuda, guía o colaboración con los adultos o de sus compañeros siempre será mayor que el nivel que pueda alcanzar por sí sólo, por lo tanto, el desarrollo cognitivo completo requiere del uso de herramientas psicológicas. Los altos porcentajes obtenidos en los ítems de la encuesta de autoevaluación y de la observación de los videos de las sesiones didácticas muestran que los docentes hacen uso de las herramientas psicológicas porque promueven una comunicación empática, a su vez realizan una reflexión crítica y autocrítica para efectuar un aprendizaje significativo con actitud crítica y finalmente existe una evaluación integral.

Otro elemento fundamental de la concepción de Vygotsky es la interacción social como proceso de desarrollo, desempeñando un papel formador y constructor. Ello significa simplemente que algunas categorías de las funciones mentales superiores (atención voluntaria, memoria lógica, pensamiento verbal y conceptual, emociones complejas, etc.) no podrían surgir y constituirse en el proceso del desarrollo sin la contribución constructora de las interacciones sociales. También se han encontrado porcentajes altos en los ítems relacionados a este elemento, tanto en la encuesta de autoevalución, así como en la observación de los videos de las sesiones de didácticas.

Tanto en la encuesta de autoevaluación como en la observación de los videos de las sesiones didácticas 
se han encontrado altos porcentajes en los ítems relacionados a los estándares intelectuales lo cual refleja que los docentes hacen uso de esta categoría porque los contenidos son en base a la interculturalidad; luego el docente contrasta crítica y autocráticamente el planteamiento de los estudiantes para luego transferir los aprendizajes a contextos socioculturales. A su vez el pensamiento crítico se propone analizar o evaluar la estructura y consistencia de los razonamientos, particularmente opiniones o afirmaciones que la gente acepta como verdaderas en el contexto de la vida sociocultural.

En cuanto a los altos porcentajes obtenidos en los ítems relacionados al uso de las destrezas intra e interpsicológicas, tanto en la encuesta de autoevaluación como en la ficha de observación de los videos de las sesiones didácticas ponen de manifiesto el uso de los docentes de esta categoría; la intención del docente se enfoca en promover el pensamiento crítico seleccionando contenidos de orden conceptual, actitudinal y procedimental en un contexto socia cultural. Asimismo, los docentes deben plantear preguntas que reflejen el pensamiento crítico, preguntas que responsabilicen por su proceso de pensamiento, preguntas que, por su uso consistente por parte del maestro en la clase, sean interiorizadas por los estudiantes como preguntas que deben plantearse ellos mismos.

Por último, también se encontró altos porcentajes en los ítems de los dos instrumentos relacionados al uso de los elementos del pensamiento, poniendo de manifiesto que los docentes consideran las opiniones de los estudiantes en un espacio de reflexión crítica y autocrítica para la toma de decisiones y resuelven problemas a partir de los conflictos cognitivos de sus estudiantes, a su vez toda verdadera enseñanza debe incluir la formación del pensamiento crítico, que aspire a favorecer el desarrollo de la autonomía.

\section{Conclusiones}

Los resultados de la investigación efectuada en una muestra de 8 docentes del Instituto Superior Pedagógico Público de Huancavelica, a los cuales se les aplicó dos intrumentos relacionados con la teoría de Vygotsky y el Pensamiento Crítico, demuestran que el nivel de pensamiento crítico de los docentes según la ficha de autoevaluación se encuentra entre bueno y óptimo, mientras que, en la ficha de observación de los videos, se encuentra entre aceptable y bueno. Esto nos permite afirmar que los docentes investigados manifiestan un pensamiento crítico por lo menos aceptable en relación a las características encontradas según la Teoría de Vygotsky.

Las características más resaltantes de la Teoría Sociocultural de Vygotsky que se manifiestan en los docentes investigados del Instituto Superior Pedagógico Público de Huancavelica son: El contexto socio-cultural, la interacción social y el uso de las herramientas psicológicas.

Los estándares intelectuales, los elementos del pensamiento y las destrezas intra e interpersonales constituyen las categorías del pensamiento crítico que se manifiestan en los docentes del Instituto Superior Pedagógico Público de Huancavelica.

Existe relación directa entre los puntajes obtenidos en los ítems sobre la teoría de Vygotsky y los obtenidos para el Pensamiento Crítico. Por lo tanto las características de la teoría socio cultural de Vygotsky encontradas se relacionan con las categorías del Pensamiento crítico que se manifiestan en los docentes del Instituto Superior Pedagógico Público de Huancavelica

\section{Agradecimientos}

A los docentes del Instituto Superior Pedagógico Público de Huancavelica por su desinteresada colaboración en la realización de este trabajo.

\section{Referencias}

[1] M. Palacios y M. Paiba, Consideraciones para una política de Desarrollo Magisterial. (Foro Educativo, Lima, Perú, 1997), pp. 25 - 43

[2] L.S. Vygotsky, El desarrollo de los procesos psicológicos superiores. (Grijalbo; México, 1988), pp. $28-87$

[3] L.S. Vygotsky, Problema del Desarrollo de la Psique. (Edit. Pedagógica, Moscú,1983), pp. 44

[4] J. Delors, La educación encierra un tesoro. Barcelona. (Grupo Santillana, Informe de la UNESCO de la Comisión Internacional sobre la Educación para el Siglo XXI, Barcelona, 1996), pp. 59

[5] J. Boisvert, La Formación del Pensamiento Crítico. (Fondo de Cultura Económica, México, 2004), pp. 48

E-mail: zoramanrique@hotmail.com 